\title{
SEMI-ONLINE PREEMPTIVE SCHEDULING: ONE ALGORITHM FOR ALL VARIANTS
}

\author{
TOMÁŠ EBENLENDR ${ }^{1}$ AND JIŘÍ SGALL ${ }^{1}$ \\ 1 Institute of Mathematics, AS CR, Žitná 25, CZ-11567 Praha 1, Czech Republic. \\ E-mail address: $\{$ ebik, sgall\}@math.cas.cz
}

\begin{abstract}
We present a unified optimal semi-online algorithm for preemptive scheduling on uniformly related machines with the objective to minimize the makespan. This algorithm works for all types of semi-online restrictions, including the ones studied before, like sorted (decreasing) jobs, known sum of processing times, known maximal processing time, their combinations, and so on. Based on the analysis of this algorithm, we derive some global relations between various semi-online restrictions and tight bounds on the approximation ratios for a small number of machines.
\end{abstract}

\section{Introduction}

We study online scheduling on uniformly related machines, which means that the time needed to process a job with processing time $p$ on a machine with speed $s$ is $p / s$. Preemption is allowed, i.e., each job may be divided into several pieces, which can be assigned to different machines in disjoint time slots. The objective is to minimize the makespan, i.e., the length of a schedule. In the online problem, jobs arrive one-by-one and we need to assign each incoming job without any knowledge of the jobs that arrive later. When a job arrives, its assignment at all times must be given and we are not allowed to change this assignment later. In other words, the online nature of the problem is given by the ordering of the input sequence and it is not related to possible preemptions and the time in the schedule.

We focus on semi-online algorithms. This term encompasses algorithms that are essentially online, but some partial information about the input is given to the scheduler in advance. The main motivation behind this approach is the observation that the classical competitive analysis is too pessimistic compared to practical results, or, in other words, the adversary who may arbitrarily determine the input sequence is too powerful. In practice, the inputs are not completely arbitrary, and it may be reasonable to restrict the set of inputs. In scheduling, numerous semi-online models have been studied; typical examples include (sequences of) jobs with decreasing processing times, jobs with bounded processing times, sequences with known total processing time of jobs and so on. Most of these models can be viewed as online algorithms on a restricted set of input sequences. Restrictions of

Key words and phrases: On-line algorithms, scheduling.

Partially supported by Institutional Research Plan No. AV0Z10190503, by Inst. for Theor. Comp. Sci., Prague (project 1M0545 of MŠMT ČR) and grant IAA1019401 of GA AV ČR.

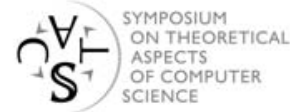

C) T. Ebenlendr and J. Sgall

(c) Creative Commons Attribution-NoDerivs License 
this type have been studied also for other online problems; the most prominent example is paging with locality of reference [1].

\section{Our results}

We give a semi-online algorithm for preemptive scheduling on uniformly related machines which is optimal for any chosen semi-online restriction, see Section 2. This means not only the cases listed above - the restriction can be given as an arbitrary set of sequences that are allowed as inputs. For any semi-online restriction, the algorithm achieves the best possible approximation ratio for any number of machines and any particular combination of machine speeds; it is deterministic, but its approximation ratio matches the best possible approximation ratio of any randomized algorithm. This generalizes and unifies previous results for various special cases of semi-online preemptive scheduling. We find such a general result providing a provably optimal algorithm for many problems quite exceptional not only in the area of scheduling but also in the whole area of online algorithms. Our result also provides a clear separation between the design of the algorithm and the analysis of the optimal approximation ratio. While the algorithm is always the same, analysis of the optimal ratio depends on the studied restrictions. Nevertheless, the general result also provides crucial new insights and methods and thus we can analyze the optimal ratio in cases that have been out of reach with previously known techniques.

For typical semi-online restrictions, we show that the optimal ratio can be computed by linear programs (with machine speeds as parameters). Studying these linear programs allows us to progress in two directions. First, we are able to completely analyze the optimal ratio for particular cases with a small number of machines. Second, we are able to study the relations between the optimal approximation ratios for different semi-online restrictions and give some bounds for a large number of machines.

The exact analysis of special cases for a small number of machines was given in $[6,3,11]$ for various restrictions, and in many more cases for non-preemptive scheduling. Typically, these results involve similar but ad hoc algorithms and an extensive case analysis which is tedious to verify, and can be done for two uniformly related machines or for more identical machines (i.e., all speeds are equal). Using our linear programs we can calculate the ratio as a formula in terms of speeds. This is a fairly routine task which can be simplified (but not completely automated) using standard mathematical software. Once the solution is known, verification amounts to checking the given primal and dual solutions for the linear program. We give a few examples of such results in Section 3; typically the verification is quite simple for $m=3$ or $m=4$.

Another research direction is to compute, for a given semi-online restriction, the optimal approximation ratio which works for any number of machines and combination of speeds. This task appears to be much harder, and even in the online case we only know that the ratio is between 2.054 and $e \approx 2.718$; the lower bound is shown by a computer-generated hard instance with no clear structure [4]. Only for identical machines, the exact ratio for any number of machines is known (i) for the online case, where it tends to $e /(e-1) \approx 1.58[2]$, and (ii) for non-increasing processing times, where it tends to $(1+\sqrt{3}) / 2 \approx 1.366$ [13].

We are able to prove certain relations between the approximation ratios for different restrictions. Some basic restrictions form an inclusion chain: The inputs where the first job has the maximal size (which is equivalent to known maximal size) include the inputs with non-increasing processing times, which in turn include the inputs with all jobs of equal size. Typically, the hard instances have non-decreasing processing times. Thus, one 
expected result is that the restriction to non-increasing processing times gives the same approximation ratio as when all jobs have equal size, even for any particular combination of speeds. The overall approximation ratio is at most 1.52, see Section 3.3. On the other hand, for known maximal size of a job we have a computer-generated hard instance with approximation ratio 1.88 with $m=120 .{ }^{1}$ Thus restricting the jobs to be non-increasing helps the algorithm much more than just knowing the maximal size of a job. This is very different from identical machines, where knowing the maximal size is equally powerful as knowing that all the jobs are equal, see [13].

More interestingly, the overall approximation ratio with known sum of processing times is the same as in the online case - even though for a small fixed number of machines knowing the sum provides a significant advantage. This is shown by a padding argument, see Section 3.1. In fact this is true also in presence of any additional restriction that allows scaling input sequences, taking a prefix, and extending the input by small jobs at the end. Thus, for example, the overall approximation ratio with non-increasing jobs and known sum of processing times is at least 1.366, using the bound for identical machines from [13].

Due to the space limit, some technical parts are available only in the extended version at http://www.math.cas.cz/ sgall/ps/semirel.pdf

\section{Preliminaries}

Let $M_{i}, i=1,2, \ldots, m$, denote the $m$ machines, and let $s_{i}$ be the speed of $M_{i}$. The machines are sorted by decreasing speeds, i.e., $s_{1} \geq s_{2} \geq \cdots \geq s_{m}$. We assume that $s_{1}>0$. The vector of speeds is denoted $\mathbf{s}$, the sum of the speeds is $S=\sum_{i=1}^{m} s_{i}$ and $S_{k}=\sum_{i=1}^{k} s_{i}$ is the sum of the $k$ largest speeds. W.l.o.g., we add infinitely many machines of speed zero, i.e., we put $s_{i}=0$ for any $i>m$. (Scheduling a job on one of these zero-speed machines means that we do not process the job at the given time at all.) Let $\mathcal{J}=\left(p_{j}\right)_{j=1}^{n}$ denote the input sequence of jobs, where $n$ is the number of jobs and $p_{j} \geq 0$ is the size, or the processing time, of the $j$ th job. The sum of processing times is denoted $P=P(\mathcal{J})=\sum_{j=1}^{n} p_{j}$. Given $\mathcal{J}$ and $i \leq n$, let $\mathcal{J}_{[i]}$ be the prefix of $\mathcal{J}$ obtained by taking the first $i$ jobs.

The time needed to process a job $p_{j}$ on machine $M_{i}$ is $p_{j} / s_{i}$; each machine can process at most one job at any time. Preemption is allowed, which means that each job may be divided into several pieces, which can be assigned to different machines, but any two time slots to which a single job is assigned must be disjoint (no parallel processing of a job); there is no additional cost for preemptions. Formally, if $t_{i}$ denotes the total length of the time intervals when the job $p_{j}$ is assigned to machine $M_{i}$, it is required that $t_{1} s_{1}+t_{2} s_{3}+\cdots+t_{m} s_{m}=p_{j}$. (A job may be scheduled in several time slots on the same machine, and there may be times when a partially processed job is not running at all.) In the (semi-)online version of this problem, jobs arrive one-by-one and at that time the algorithm has to give a complete assignment of this job at all times, without the knowledge of the jobs that arrive later. The objective is to find a schedule of all jobs in which the maximal completion time (the makespan) is minimized.

For an algorithm $\mathrm{A}$, let $C_{\max }^{\mathrm{A}}[\mathcal{J}]$ be the makespan of the schedule of $\mathcal{J}$ produced by A. By $C_{\max }^{*}[\mathcal{J}]$ we denote the makespan of the optimal offline schedule of $\mathcal{J}$. A (randomized) algorithm $\mathrm{A}$ is an $R$-approximation if for every input $\mathcal{J}$, the (expected) makespan is at most $R$ times the optimal makespan, i.e., $\mathbb{E}\left[C_{\max }^{\mathrm{A}}[\mathcal{J}]\right] \leq R \cdot C_{\max }^{*}[\mathcal{J}]$.

The optimal makespan can be computed as $C_{\max }^{*}[\mathcal{J}]=\max \left\{P / S, \max _{k=1}^{m-1}\left\{P_{k} / S_{k}\right\}\right\}$, where $P_{k}$ denotes the sum of the $k$ largest processing times in $\mathcal{J}$ and $S_{k}$ is the sum of the $k$

\footnotetext{
${ }^{1}$ See the Maple output at http://www.math.cas.cz/ sgall/ps/semirel-pmax.mpl
} 
largest speeds. This is a lower bound on the makespan, as the first term gives the minimal time when all the work can be completed using all the machines fully, and similarly the term for $k$ is the minimal time when the work of the $k$ largest jobs can be completed using the $k$ fastest machines fully. The tightness of this bound follows from [10, 8, 5].

\section{Semi-online restrictions and previous work}

We define a general semi-online input restriction to be simply a set $\Psi$ of allowed inputs, also called input sequences. We call a sequence a partial input if it is a prefix of some input sequence; the set of all partial inputs is denoted $\operatorname{pref}(\Psi)$. Thus the partial inputs are exactly the sequences that the algorithm can see at some point. A (randomized) semi-online algorithm $\mathrm{A}$ with restriction $\Psi$ is an $R$-approximation algorithm if $\mathbb{E}\left[C_{\max }^{\mathrm{A}}[\mathcal{J}]\right] \leq R \cdot C_{\max }^{*}[\mathcal{J}]$ for any $\mathcal{J} \in \Psi$. Note that this implies that for any prefix $\mathcal{J}^{\prime}$ of $\mathcal{J}, \mathbb{E}\left[C_{\max }^{\mathrm{A}}\left[\mathcal{J}^{\prime}\right]\right] \leq R \cdot C_{\max }^{*}[\mathcal{J}]$.

Below we list some of the restrictions that are studied in the literature, together with the notation that we are going to use, the previous work, and our results.

Known sum of processing times, $\sum p_{j}=P$. For a given value $\bar{P}, \Psi$ contains all sequences with $P=\bar{P}$. We prove that the overall ratio is surprisingly the same as in the general online case, on the other hand we note that for $m=2$, 1-approximation is possible and we analyze the cases of $m=3,4$.

Non-increasing processing times, denoted decr. Here $\Psi$ contains all sequences with $p_{1} \geq p_{2} \geq \cdots \geq p_{n}$. For $m=2$, the optimal algorithm for all $\mathbf{s}$ was analyzed in [6] and for identical machines in [13]. We prove that for any $\mathbf{s}$ this case is the same as the case with all jobs equal. We analyze the cases for $m=2,3$, and prove some bounds for larger $m$.

Known optimal makespan, $C_{\max }^{*}=T$. For a given value $\bar{T}, \Psi$ contains all sequences with $C_{\max }^{*}[\mathcal{J}]=\bar{T}$. A 1-approximation semi-online algorithm is known for any s, see [5].

Known maximal job size, $p_{\max }=p$. For a given value $\bar{p}, \Psi$ contains all sequences with $\max p_{j}=\bar{p}$. This is equivalent to the case when the first job is maximal, as any algorithm for that special case can be used also for the case when the maximal job arrives later. Thus this restriction also includes non-increasing jobs. In [13] it is shown that for identical machines, the approximation ratio is the same as when the jobs are non-increasing. We show that this is not the case for general speeds. This restriction was introduced in [9] for non-preemptive scheduling on 2 identical machines.

Tightly grouped processing times. For given values $\bar{p}$ and $\alpha, \Psi$ contains all sequences with $p_{j} \in[\bar{p}, \alpha \bar{p}]$ for each $j$. This restriction was introduced in [9] for non-preemptive scheduling on 2 identical machines. Tight bounds for preemptive scheduling on 2 uniformly related machines were given in [3].

Inexact partial information. In this case, some of the previously considered values (optimal makespan, sum of job sizes, maximal job size) is not known exactly but only up to a certain factor. These variants were studied first in [15] without preemption and then in [11] for preemptive scheduling; both on identical machines.

Online scheduling. Here $\Psi$ contains all sequences. In our (i.e., the authors and Wojtek Jawor) previous work [4], we have designed an optimal online algorithm for all speed vectors. The algorithm and the proof of the main result in this paper generalize that result, using the same techniques, however, some technical issues have to be handled carefully to achieve the full generality of our new result. Online preemptive scheduling was studied first in [2].

The paper [12] is probably the first paper which studied and compared several notions of semi-online algorithms, including known sum of processing times. Some combination 
of the previous restrictions were studied in [14] for non-preemptive scheduling on identical machines. We should note that there are also semi-online models that do not fit into our framework at all. For example, the algorithm may get a hint which job is the last one, or it is allowed to store some job(s) in a buffer.

\section{The optimal algorithm}

The new algorithm is based on the algorithm for online scheduling from [4]. In this section we present the key ideas with emphasis on the issues that need to be handled differently in the more general semi-online setting.

Suppose that we are given a parameter $r$ and we try to develop an $r$-approximation algorithm. In the online case, we simply make sure that the current job completes by time $r$ times the current optimal makespan. In the semi-online case, if the restriction is not closed under taking a prefix, this would be too pessimistic. It may happen that the current partial input is not in $\Psi$ and we know that any extension in $\Psi$ has much larger optimal makespan (e.g., if the restriction forces that some large jobs will arrive later). In this case we can schedule the current job so that it complete much later than at time $r$ times the current optimal makespan. For this purpose, we define the appropriate quantity to be used instead of the current optimal makespan.

Definition 2.1. For an input restriction $\Psi$ and a partial input $\mathcal{I} \in \operatorname{pref}(\Psi)$, we define the optimal makespan as the infimum over all possible end extensions of $\mathcal{J}$ that satisfy $\Psi$ :

$$
C_{\max }^{*, \Psi}[\mathcal{I}]=\inf \left\{C_{\max }^{*}[\mathcal{J}] \mid \mathcal{J} \in \Psi \& \mathcal{I} \text { is a prefix of } \mathcal{J}\right\}
$$

Note that for any input sequence $\mathcal{J} \in \Psi$ we have $C_{\max }^{*}[\mathcal{J}]=C_{\max }^{*, \Psi}[\mathcal{J}]$.

\section{Algorithm RatioStretch}

Our algorithm takes as a parameter a number $r$ which is the desired approximation ratio. Later we show that, for the right choice of $r$, our algorithm is optimal. Given $r$, we want to schedule each incoming job so that it completes at time $r \cdot C_{\max }^{*, \Psi}\left[\mathcal{J}_{[j]}\right]$. By the definition of $C_{\max }^{*, \Psi}\left[\mathcal{J}_{[j]}\right]$, any schedule for any possible extension of the current partial input

will have makespan at least $C_{\max }^{*, \Psi}\left[\mathcal{J}_{[j]}\right]$, in particular $C_{\max }^{*, \Psi}\left[\mathcal{J}_{[j]}\right] \leq C_{\max }^{*}[\mathcal{J}]$. Thus, if each job $j$ completes by time $r \cdot C_{\max }^{*, \Psi}\left[\mathcal{J}_{[j]}\right] \leq r \cdot C_{\max }^{*}[\mathcal{J}]$, we have an $r$-approximation algorithm.

Even when we decide the completion time of a job, there are many ways to schedule it given the flexibility of preemptions. We choose a particular one based on the notion of a virtual machine from $[5,4]$. We define the $i$ th virtual machine, denoted $V_{i}$, so that at each time $\tau$ it contains the $i$ th fastest machine among those real machines $M_{1}, M_{2}, \ldots, M_{m}$ that are idle at time $\tau$. Due to preemptions, a virtual machine can be thought and used as a single machine with changing speed. When we schedule (a part of) a job on a virtual machine during some interval, we actually schedule it on the corresponding real machines that are uniquely defined at each time.

Upon arrival of a job $j$ we compute a value $T_{j}$ defined as $r \cdot C_{\max }^{*, \Psi}\left[\mathcal{J}_{[j]}\right]$. Then we find two adjacent virtual machines $V_{k}$ and $V_{k+1}$, and time $t_{j}$, such that if we schedule $j$ on $V_{k+1}$ in the time interval $\left(0, t_{j}\right]$ and on $V_{k}$ from $t_{j}$ on, then $j$ finishes exactly at time $T_{j}$.

We need to show that we can always find such machines $V_{k}$ and $V_{k+1}$. Since we have added the machines of speed 0 , it only remains to prove that each job can fit on $V_{1}$. This is true for the appropriate value of $r$. 
Before we sketch the proof, we make a few remarks concerning efficiency and uniformity of the algorithm. The only parts of the algorithm that depend on the semi-online restriction are (i) the computation of the optimal approximation ratio and (ii) the computation of $C_{\max }^{*, \Psi}[\mathcal{J}]$. The rest of the algorithm is independent of the restriction and very efficient. Similarly to the online algorithms, for semi-online algorithms we generally do not require the computation to be polynomial time. For a general restriction the optimal algorithm cannot be efficient. (If the set of input sequences is, e.g., not recursive, then it may be algorithmically undecidable how much time we have even for scheduling the first job. Besides, there are more possible restrictions than algorithms.) Nevertheless, the algorithm is efficient for many natural restrictions. Computing $\left.C_{\max }^{*} \Psi \mathcal{J}\right]$ is usually simple. If the restriction is closed under taking prefixes, then it is equal to $C_{\max }^{*}[\mathcal{J}]$. In other cases it is easy to see which extension has the smallest makespan. Computing the optimal approximation ratio is more difficult, but in Section 3 it is shown that in many natural cases it reduces to linear programming. Alternatively, we can use any upper bound on the approximation ratio and give to the algorithm as a parameter.

\section{Optimality of Algorithm RatioStretch}

Our goal is to show that Algorithm RatioStretch works whenever the parameter $r$ is at least the optimal approximation ratio for the given $\Psi$ and $\mathbf{s}$. We actually prove the converse: Whenever for some input $\mathcal{J}$ Algorithm RatioStretch with the parameter $r$ fails, we prove that there is no $r$-approximation algorithm.

This is based on a generalization of a lemma from [7] which provides the optimal lower bounds for online algorithms, as shown in [4]. The key observation in its proof is this: On an input $\mathcal{J}$, if the adversary stops the input sequence at the $i$ th job from the end, any $r$ competitive online algorithm must complete by time $r$ times the current optimal makespan, and after this time, in the schedule of $\mathcal{J}$, only $i-1$ machines can be used. This bounds the total work of all the jobs in terms of $r$ and optimal makespans of the prefixes, and thus gives a lower bound on $r$. To generalize to an arbitrary restriction $\Psi$, we need to deal with two issues.

First, the adversary cannot stop the input if the current partial input is not in $\Psi$. Instead, the sequence then must continue so that its optimal makespan is the current $C_{\max }^{*, \Psi}$ (or its approximation). Consequently, the bound obtained uses $C_{\max }^{*, \Psi}$ in place of previous $C_{\max }^{*}$, which possibly decreases the obtained bound.

Second, for a general semi-online restriction, using the last $m$ prefixes of $\mathcal{J}$ may not give the best possible lower bound. E.g., the restriction may force that some job is tiny, and thus using the prefix ending at this job is useless; in general, we also cannot remove such a job from the input sequence. To get a stronger lower bound, we choose a subsequence of important jobs from $\mathcal{J}$ and bound their total work in terms of values $C_{\max }^{*, \Psi}$ of the prefixes of the original sequence $\mathcal{J}$.

Lemma 2.2. Let $\mathbf{A}$ be any randomized $R$-approximation semi-online algorithm for preemptive scheduling on $m$ machines with an input restriction $\Psi$. Then for any partial input $\mathcal{J} \in \operatorname{pref}(\Psi)$, for any $k$, and for any subsequence of jobs $1 \leq j_{1}<j_{2}<\cdots<j_{k} \leq n$ we have

$$
\sum_{i=1}^{k} p_{j_{i}} \leq R \cdot \sum_{i=1}^{k} s_{k+1-i} C_{\max }^{*, \Psi}\left[\mathcal{J}_{\left[j_{i}\right]}\right]
$$


Let $r^{\Psi}$ be the largest lower bound on the approximation ratio obtained by Lemma 2.2:

Definition 2.3. For any vector of speeds $\mathbf{s}$ and any partial input $\mathcal{J} \in \operatorname{pref}(\Psi)$,

$$
r^{\Psi}(\mathbf{s}, \mathcal{J})=\sup _{1 \leq j_{1}<j_{2}<\cdots<j_{k} \leq n} \frac{\sum_{i=1}^{k} p_{j_{i}}}{\sum_{i=1}^{k} s_{k+1-i} \cdot C_{\max }^{*,}\left[\mathcal{J}_{\left[j_{i}\right]}\right]} .
$$

For any $\mathbf{s}$, let $r^{\Psi}(\mathbf{s})=\sup _{\mathcal{J} \in \operatorname{pref}(\Psi)} r^{\Psi}(\mathbf{s}, \mathcal{J})$. Finally, let $r^{\Psi}=\sup _{\mathbf{s}} r^{\Psi}(\mathbf{s})$.

With these definitions and Lemma 2.2, we can prove the following main theorem. If Algorithm RatioStretch cannot schedule the incoming job, we choose a subsequence including the jobs scheduled so far on the first virtual machine and the incoming job. We use Lemma 2.2 with this subsequence to argue that that no (randomized) algorithm can have the same approximation ratio.

Theorem 2.4. For any restriction $\Psi$ and vector of speeds $\mathbf{s}$, Algorithm RatioStretch with a parameter $r \geq r^{\Psi}(\mathbf{s})$ is an $r$-approximation algorithm for semi-online preemptive scheduling on $m$ uniformly related machines. In particular, $r^{\Psi}(\mathbf{s})$ (resp. $\left.r^{\Psi}\right)$ is the optimal approximation ratio for semi-online algorithms for $\Psi$ with speeds $\mathbf{s}$ (resp. with arbitrary speeds).

\section{Reductions and linear programs}

We have a formula for $r^{\Psi}(\mathbf{s})$ which gives the desired approximation ratio for any speeds and $\Psi$ as a supremum over a bound for all partial inputs and all their subsequences. It is not obvious how to turn this into an efficient algorithm. Now we develop a general methodology how to compute the ratio using linear programs and apply it to a few cases.

We observed that for a general restriction it may be necessary to use an arbitrary subsequence in Definition 2.3. However, for many restrictions it is sufficient to use the whole sequence, similarly as for online scheduling. Usual restrictions are essentially of two kinds. The first type are the restrictions that put conditions on individual jobs or their order. These restrictions are closed under taking subsequences (not only prefixes), i.e., any subsequence of an input sequence is also in $\Psi$. The second type are the restrictions where some global information is given in advance, like $\sum p_{j}=P$ or $C_{\max }^{*}=T$. These are not closed under taking subsequences, but are closed under permuting the input sequence.

We define a large class of restrictions that includes both types of restrictions discussed above as well as their combinations; in particular it includes all the restrictions listed and studied here. The definition below implies that any subsequence of any input sequence is a prefix of another input. Thus, the sets of all the subsequences and all the prefixes of $\Psi$ coincide, and Definition 2.3 simplifies using the monotonicity condition in the definition.

Definition 3.1. An input restriction $\Psi$ is proper if for any $\mathcal{J} \in \Psi$ and any subsequence $\mathcal{I}$ of $\mathcal{J}$, we have $\mathcal{I} \in \operatorname{pref}(\Psi)$ and furthermore $C_{\max }^{*, \Psi}[\mathcal{I}] \leq C_{\max }^{*, \Psi}[\mathcal{J}]$.

Definition 3.2. Let $\Psi$ be a proper semi-online restriction and $\mathcal{J} \in \operatorname{pref}(\Psi)$ a partial input. We define

$$
\bar{r}^{\Psi}(\mathbf{s}, \mathcal{J})=\frac{\sum_{j=1}^{n} p_{j}}{\sum_{j=1}^{n} s_{n+1-j} \cdot C_{\max }^{*, \Psi}\left[\mathcal{J}_{[j]}\right]} .
$$


From now on, we focus on proper restrictions. It may happen that $r^{\Psi}(\mathbf{s}, \mathcal{J})>\bar{r}^{\Psi}(\mathbf{s}, \mathcal{J})$. By Definitions 2.3 and 2.3 we may take a subsequence of jobs $\mathcal{I}=\left(p_{j_{i}}\right)_{i=1}^{k}$ that achieves the value of $\bar{r}^{\Psi}(\mathbf{s}, \mathcal{I}) \geq r^{\Psi}(\mathbf{s}, \mathcal{J})-\varepsilon$ for any $\varepsilon>0$. By the definition of a proper restriction, $\mathcal{I} \in \operatorname{pref}(\Psi)$. Taking the supremum over all partial inputs, we obtain the following simpler formula for the optimal approximation ratio.

Observation 3.3. For any proper restriction $\Psi, r^{\Psi}(\mathbf{s})=\sup _{\mathcal{J} \in \operatorname{pref}(\Psi)} \bar{r}^{\Psi}(\mathbf{s}, \mathcal{J})$.

Our strategy is to reduce the number of sequences $\mathcal{J}$ that need to be taken into account. Typically, we show that the sequences must be sorted. Then we know which jobs are the biggest ones and we can express the optimal makespans for prefixes by linear constraints in job sizes. Maximizing the expression for $\bar{r}^{\Psi}(\mathbf{s})$, which gives the desired bound, is then reduced to solving one or several linear programs. The following observation helps us to limit the set of relevant sequences.

Observation 3.4. Let $\Psi$ be arbitrary proper restriction, let $\mathbf{s}$ be arbitrary speed vector, and let $\mathcal{J}, \mathcal{J}^{\prime} \in \operatorname{pref}(\Psi)$,. be two partial inputs with $n$ jobs. Suppose that for some $b>0$ :

$$
\begin{aligned}
\sum_{j=1}^{n} p_{j}^{\prime} & =b \cdot \sum_{j=1}^{n} p_{j}, \quad \text { and } \\
(\forall i=1, \ldots, n) \quad C_{\max }^{*, \Psi}\left[\mathcal{J}_{[i]}^{\prime}\right] & \leq b \cdot C_{\max }^{*, \Psi}\left[\mathcal{J}_{[i]}\right] .
\end{aligned}
$$

Then $\bar{r}\left(\mathbf{s}, \mathcal{J}^{\prime}\right) \geq \bar{r}(\mathbf{s}, \mathcal{J})$.

The observation follows immediately from the definition of $\bar{r}^{\Psi}(\mathbf{s}, \mathcal{J})$.

Whenever (i) $\Psi$ is closed under permutations of the sequence and (ii) increasing the size of the last job of a partial input cannot decrease $C_{\max }^{*, \Psi}$, the observation implies that it is sufficient to consider sequences of non-decreasing jobs: If $\mathcal{J}$ contains two jobs with $p_{k}<p_{k+1}$, swapping them can only increase $C_{\max }^{*, \Psi}\left[\mathcal{J}_{[k]}\right]$ and any other $C_{\max }^{*, \Psi}\left[\mathcal{J}_{[i]}\right]$ remains unchanged; thus the observation applies with $b=1$.

\subsection{Known sum of processing times, $\sum p_{j}=P$}

Here we are given a value $\bar{P}$ and $\Psi$ contains all $\mathcal{J}$ with $P=\bar{P}$. It can be easily verified that $C_{\max }^{*, \Psi}[\mathcal{J}]=\max \left\{C_{\max }^{*}[\mathcal{J}], \bar{P} / S\right\}$ for any $\mathcal{J}$ with $P \leq \bar{P}$.

Since we can permute the jobs and increasing the size of the last job does not decrease $C_{\max }^{*, \Psi}$, Observation 3.4 implies that we can restrict ourselves to non-decreasing sequences $\mathcal{J}$. Furthermore, we may assume that $P=\bar{P}$ : We know that $P \leq \bar{P}$, as otherwise $\mathcal{J}$ is not a partial input. If $P<\bar{P}$, we scale up $\mathcal{J}$ to $\mathcal{J}^{\prime}$ by multiplying all the sizes by $b=P^{\prime} / P$. Observation 3.4 then applies, as each $C_{\max }^{*, \Psi}\left[\mathcal{J}_{[i]}^{\prime}\right]=\max \left\{C_{\max }^{*}\left[\mathcal{J}_{[i]}^{\prime}\right], \bar{P} / S\right\}$ increases by at most the scaling factor $b$. Finally, we observe that we can restrict ourselves to sequences $\mathcal{J}$ with less than $m$ jobs. If $n \geq m$, we use the fact that $C_{\max }^{*, \Psi}\left[\mathcal{J}_{[i]}\right] \geq \bar{P} / S$ for any $i$ and obtain $\bar{r}^{\Psi}(\mathbf{s}, \mathcal{J})=P /\left(\sum_{i=1}^{n} s_{n+1-i} \cdot C_{\max }^{*}\left[\mathcal{J}_{[i]}\right]\right) \leq P /\left(\sum_{i=1}^{n} s_{n+1-i} \cdot \bar{P} / S\right)=1$, using $n \geq m$ in the last step.

Summarizing, we can assume that $\mathcal{J}$ is a non-decreasing sequence of $n<m$ jobs with $P=\bar{P}$. (Note that this does not mean that the adversary uses fewer jobs than machines, as he may need to release some small jobs at the end of the prefix sequence, to extend it to a sequence in $\Psi$.) To obtain the worst case bound, we compute $m-1$ linear programs, 
one for each value of $n$, and take the maximum of their solutions. The linear program for a given $P$, s, and $n$ has variables $q_{i}$ for job sizes and $O_{i}$ for optimal makespans of the prefixes:

$$
\begin{aligned}
& \text { minimize } \quad r^{-1}=\frac{s_{1} O_{n}+s_{2} O_{n-1}+\cdots+s_{n} O_{1}}{\bar{P}} \\
& \text { subject to } \\
& q_{1}+\cdots+q_{n}=\bar{P} \\
& \bar{P} \leq\left(s_{1}+s_{2}+\cdots+s_{m}\right) O_{k} \quad \text { for } k=1, \ldots, n \\
& q_{j}+q_{j+1}+\cdots+q_{k} \leq\left(s_{1}+s_{2}+\cdots+s_{k-j+1}\right) O_{k} \quad \text { for } 1 \leq j \leq k \leq n \\
& 0 \leq q_{j} \leq q_{j+1} \quad \text { for } j=1, \cdots, n-1
\end{aligned}
$$

If we fix the input sequence, i.e., the values of $q_{i}$, then the smallest objective is achieved for $O_{k}$ as small as possible which is exactly the value of the optimal makespan, by the constraints involving $O_{k}$. Thus the linear program computes correctly the value $1 / r^{\sum p_{j}=P}(\mathbf{s})$. We can also see that the linear program scales and the optimum does not depend on $\bar{P}$.

We now examine the special cases of $m=2,3$. The linear program is trivial for $n=1$, and we conclude that for $m=2$ the approximation ratio is equal to 1, i.e., RatioStretch always produces an optimal schedule. We can see this also intuitively: The algorithm starts scheduling the incoming jobs in the interval $\left[0, T_{1}\right)$ where $T_{1} \geq \bar{P} / S$. Consider the first time when a job is scheduled at the first real machine $M_{1}$. It is always possible to schedule this job at the empty machine $M_{1}$ so that it completes before the current optimal makespan. Furthermore, after $M_{1}$ is used the first time, the algorithm guarantees that in the interval $\left[0, T_{1}\right)$ there is only one real machine idle at any time. This in turn implies that the remaining jobs can be completed by time $T_{1}$, as the total size of all jobs is $\bar{P} \leq S \cdot T_{1}$.

For $m=3$, it remains to solve the linear program for $n=2$. The resulting ratio is:

$$
r^{\sum p_{j}=P}\left(s_{1}, s_{2}, s_{3}\right)= \begin{cases}\frac{s_{1}\left(s_{1}+s_{2}\right)}{s_{1}^{2}+s_{2}^{2}} & \text { for } s_{1}^{2} \leq s_{2}\left(s_{2}+s_{3}\right) \\ 1+\frac{s_{2}}{s_{1}\left(s_{1}+s_{2}+s_{3}\right)+s_{2}\left(s_{1}+s_{2}\right)} & \text { for } s_{1}^{2} \geq s_{2}\left(s_{2}+s_{3}\right)\end{cases}
$$

The overall worst case ratio for three machines is $\frac{2+\sqrt{2}}{3} \approx 1.138$ for $s_{1}=\sqrt{2}, s_{2}=s_{3}=1$.

Padding. We prove a theorem that shows that knowing the total size of jobs does not improve the overall approximation ratio. This may sound surprising, as for two machines, knowing the sum allows to generate an optimal schedule, and also for three machines the improvement is significant. The same result holds also in presence of an additional restriction with suitable properties. Among the restrictions that we consider, the requirements are satisfied for non-increasing jobs, known maximal job size, or the online case. By " $\Psi, \sum p_{j}=P$ " we denote the intersection of the two restrictions, i.e., the set of all sequences $\left(p_{j}\right)_{j=1}^{n} \in \Psi$ such that $\sum_{i=1}^{n} p_{j}=\bar{P}$ for a given value of $\bar{P}$

We say that $\Psi$ allows scaling if for any $\mathcal{J} \in \Psi$ and $b>0$, the modified sequence $\mathcal{J}^{\prime}=\left(b p_{j}\right)_{j=1}^{n}$ satisfies $\mathcal{J}^{\prime} \in \Psi$. We say that $\Psi$ allows padding if for any $\mathcal{J} \in \Psi$, there exists $\varepsilon_{0}>0$ such that any sequence $\mathcal{J}^{\prime}$ created by extending $\mathcal{J}$ by an arbitrary number of equal jobs of size $\varepsilon<\varepsilon_{0}$ at the end satisfies $\mathcal{J}^{\prime} \in \Psi$.

Theorem 3.5. Suppose that $\Psi$ is proper, allows scaling, padding, and is closed under taking prefixes. Let $\mathcal{J} \in \Psi$ and let $\mathbf{s}$ be arbitrary. Then for any $\delta>0$ there exists $\mathcal{J}^{\prime}$ and $\mathbf{s}^{\prime}$ such that $\bar{r}^{\Psi, \sum p_{j}=P}\left(\mathbf{s}^{\prime}, \mathcal{J}^{\prime}\right) \geq \bar{r}^{\Psi}(\mathbf{s}, \mathcal{J}) /(1+\delta)$. Consequently, $r^{\Psi, \sum p_{j}=P}=r^{\Psi}$.

Proof. We fix $\mathbf{s}, \mathcal{J}$, and $\bar{P}$ given to the algorithm with the restriction $\sum p_{j}=P$. We proceed towards constructing the appropriate $\mathbf{s}^{\prime}$ and $\mathcal{J}^{\prime}$. 
Since $\Psi$ allows scaling, the value $\left.C_{\max }^{*} \Psi \mathcal{J}\right]$ is multiplied by $b$ when $\mathcal{J}$ is scaled by $b$. Consequently, the value of $\bar{r}^{\Psi}(\mathbf{s}, \mathcal{J})$ does not change when $\mathcal{J}$ is scaled. Let $\mathcal{J}^{\prime}=\left(p_{j}^{\prime}\right)_{j=1}^{n}$ be the sequence $\mathcal{J}$ scaled so that $\sum_{j=1}^{n} p_{j}^{\prime}=\bar{P}$. Then $\bar{r}^{\Psi}\left(\mathbf{s}, \mathcal{J}^{\prime}\right)=\bar{r}^{\Psi}(\mathbf{s}, \mathcal{J})$.

Choose a small $\sigma>0$ so that $\sigma<s_{m}$ and $\sigma<\delta S / n$. Let $O_{1}=p_{1}^{\prime} / s_{1}$, i.e., the optimal makespan after the first job. Let $\mathbf{s}^{\prime}$ be the sequence of speeds starting with $\mathbf{s}$ and continuing with $n+\bar{P} /\left(O_{1} \sigma\right)$ of values $\sigma$. The first condition on $\sigma$ guarantees that $\mathbf{s}^{\prime}$ is monotone and thus a valid sequence of speeds. The second condition guarantees that the added machines are sufficiently slow, so that for any sequence of at most $n$ jobs, in particular for the prefixes of $\mathcal{J}^{\prime}$, the makespan decreases by at most the factor of $(1+\delta)$. Since $\Psi$ is closed under taking prefixes, $C_{\max }^{*} \Psi$ equals $C_{\max }^{*}$ for any sequence. Thus we conclude that $\bar{r}^{\Psi}\left(\mathbf{s}^{\prime}, \mathcal{J}^{\prime}\right) \geq \bar{r}^{\Psi}\left(\mathbf{s}, \mathcal{J}^{\prime}\right) /(1+\delta)$.

Finally, we have added sufficiently many new machines so that for any sequence of at most $n$ jobs, the empty new machines can accommodate total work of $\bar{P}$ without exceeding makespan $O_{1}$. This implies that for all prefixes of $\mathcal{J}^{\prime}, C_{\max }^{*, \Psi, \sum p_{j}=P}\left[\mathcal{J}_{[i]}^{\prime}\right]=C_{\max }^{*, \Psi}\left[\mathcal{J}_{[i]}^{\prime}\right]$; thus $\bar{r} \Psi, \sum p_{j}=P\left(\mathbf{s}^{\prime}, \mathcal{J}^{\prime}\right)=\bar{r}^{\Psi}\left(\mathbf{s}^{\prime}, \mathcal{J}^{\prime}\right) \geq \bar{r}^{\Psi}\left(\mathbf{s}, \mathcal{J}^{\prime}\right) /(1+\delta) \geq \bar{r}^{\Psi}(\mathbf{s}, \mathcal{J}) /(1+\delta)$.

\subsection{Known maximal processing time, $p_{\max }=p$}

Here we are given $\bar{p}$, the maximal size of a job. As noted before, any algorithm that works with the first job being the maximal one can be easily changed to a general algorithm for this restriction. First it virtually schedules the maximal job and then it compares the size of each job to $\bar{p}$. If it is equal for the first time, it schedules the job to the time slot(s) it reserved by virtual scheduling at the beginning. Other jobs are scheduled in the same way in both algorithms. Thus we can work with the equivalent restriction containing all the sequences where the first job is maximal. Then $\left.C_{\max }^{*} \Psi \mathcal{J}\right]=C_{\max }^{*}[\mathcal{J}]$ for any partial input. By Observation 3.4, the other jobs can be reordered as in the previous case, and we can maximize only over sequences with non-decreasing job sizes from the second job on.

In this case we are able to use a single linear program to cover input sequences of an arbitrary length. The variables are: $p$ for the length of the first job, $q_{1}$ for the total length of jobs $p_{2}, \ldots, p_{n-m+1}$, and $q_{2}, \ldots, q_{m}$ for the jobs $p_{n-m+2}, \ldots, p_{n}$. For sequences with $n<m$, we set $q_{1}=q_{2}=\cdots q_{n-m}=0$. Consider the following non-linear program:

$$
\begin{array}{rll}
\text { maximize } & r=\frac{P}{s_{1} O_{m}+s_{2} O_{m-1}+\cdots+s_{m} O_{1}} \\
\text { subject to } & & \\
p+q_{1}+\cdots+q_{m} & =P & \\
p+q_{1}+\cdots+q_{k} & \leq\left(s_{1}+s_{2}+\cdots+s_{m}\right) O_{k} & \text { for } k=1, \ldots, m \\
p+q_{j+1}+q_{j+2}+\cdots+q_{k} & \leq\left(s_{1}+s_{2}+\cdots+s_{k-j+1}\right) O_{k} & \text { for } 1 \leq j \leq k \leq m \\
0 \leq q_{j} & \leq q_{j+1} \leq p & \text { for } j=2, \cdots, m-1 \\
0 & \leq q_{1} &
\end{array}
$$

If we fix the values of $q_{i}$, then the largest objective is achieved for $O_{k}$ as small as possible. By the constraints involving $O_{k}$, this is exactly the value of the optimal makespan for a sequence where $q_{1}$ represents a prefix of a sequence of jobs smaller than $q_{2}$. Thus the program computes correctly the value $r^{\sum p_{j}=P}(\mathbf{s})$. The program scales, thus we can scale any feasible solution so that the denominator of the objective function is a given constant. Thus we get an equivalent linear program after adding the constraint $1=s_{1} O_{m}+s_{2} O_{m-1}+\cdots+s_{m} O_{1}$. 
Small number of machines. For two machines we get the approximation ratio

$$
r^{p_{\max }=p}\left(s_{1}, s_{2}\right)=1+\frac{s_{1} s_{2}}{\left(s_{1}+s_{2}\right)^{2}+s_{1}^{2}}
$$

The maximum is 1.2 for $s_{1}=s_{2}$. For three machines we get

$$
r^{p_{\max }=p}\left(s_{1}, s_{2}, s_{3}\right)= \begin{cases}1+\frac{s_{1}\left(s_{2}+s_{3}\right)}{S^{2}+s_{1}^{2}} & \text { for } s_{1} s_{2} \geq s_{3} S \\ 1+\frac{s_{1} s_{2}+2 s_{1} s_{3}}{S^{2}+2 s_{1}^{2}+s_{1} s_{2}} & \text { for } s_{1} s_{2} \leq s_{3} S\end{cases}
$$

This is maximized at $s_{1}=2, s_{2}=s_{3}=\sqrt{3}$ which gives the ratio $(8+12 \sqrt{3}) / 23 \approx 1.252$.

\subsection{Non-increasing processing times, decr}

We are also interested in sequences of non-increasing jobs, as this is one of the most studied restrictions. Now $\Psi$ contains sequences which have $p_{j} \geq p_{j+1}$ for all $j$. We cannot swap jobs, however, we can take two adjacent jobs $j$ and $j+1$ and replace both of them by jobs of the average size $\left(p_{j}+p_{j+1}\right) / 2$. By Observation 3.4, the approximation ratio does not decrease. Similarly, we can replace longer segment of jobs with only two distinct sizes by the same number of jobs of the average size. Repeating this process, we can conclude that for the worst case for a given set of speeds it is sufficient to consider sequences where all jobs have equal size. By scaling, the actual size of jobs does not matter, we only need to determine the length of the sequence which gives the highest ratio.

Let us denote $\hat{r}_{n}(\mathbf{s})=\bar{r}^{\text {decr }}(\mathbf{s}, \mathcal{J})$ for a sequence $\mathcal{J}$ with $n$ jobs with $p_{j}=1$. For this sequence, $C_{\max }^{*, \Psi}[\mathcal{J}]=C_{\max }^{*}[\mathcal{J}]=n / S_{n}$. (Recall that $s_{i}=0$ for $i>m$ and $S_{k}=\sum_{i=1}^{k} s_{i}$.) Using this for the prefixes, we obtain from Observation 3.3 that

$$
\hat{r}_{n}(\mathbf{s})=n \cdot\left(\sum_{k=1}^{n} \frac{k s_{n-k+1}}{S_{k}}\right)^{-1} .
$$

It can be seen that for any speed vector, the sequence $\hat{r}_{n}(\mathbf{s})$ decreases with $n$ for $n \geq 2 m$. Thus computing the approximation ratio for any given speeds is efficient.

A natural approach to estimate the overall ratio is to find for each $n$ the worst speed vector and the corresponding ratio $\hat{r}_{n}=\sup _{\mathbf{s}} \hat{r}_{n}(\mathbf{s})$. Based on numerical experiments, we conjecture that for each $n, \hat{r}_{n}$ is attained for some $\mathbf{s}$ with $s_{1}=s_{2}=\cdots=s_{m-1}$. I.e., almost all the speeds are equal. This conjecture would imply that with non-increasing jobs, the optimal overall approximation ratio is the same for the uniformly related machines and for the identical machines, and this is equal to $(1+\sqrt{3}) / 2 \approx 1.366$ by [13].

This is related to an intriguing geometric question. Suppose we have numbers $x_{i}, y_{i}, i=$ $1, \ldots, n$ such that $x_{i} y_{i}=i$ for all $i$ and both sequences $\left(x_{i}\right)_{i=1}^{n}$ and $\left(y_{i}\right)_{i=1}^{n}$ are non-decreasing. Consider the union of rectangles $\left[0, x_{i}\right] \times\left[0, y_{n+1-i}\right]$ over all $i$; this is a staircase-like part of the positive quadrant of the plane. What is the smallest possible area of this union of rectangles? We conjecture that the minimum is attained for some $y_{1}=y_{2}=\ldots=y_{k}$ and $x_{k+1}=x_{k+2}=\ldots=x_{n}$ for some $k$. This would imply the previous conjecture.

We are not able to determine exactly the values of $\hat{r}_{n}$, but we can prove certain relations between these values. In particular, for any integers $a, n$, and $n^{\prime}, r_{a n} \geq r_{n}$ and $r_{n^{\prime}} \leq \frac{n+1}{n} r_{n}$. For the first proof, we replace a sequence of speeds from the bound for $r_{n}$ by a sequence where each speed is repeated $a$ times, and the bound follows by manipulating the formula 
for $r_{n}$. The second inequality is shown by replacing the speeds for $r_{n^{\prime}}$ by a shorter sequence where each new speed is a sum of a segment of $a$ speeds in the original sequence, for a suitable $a$. These relations show that whenever we are able to evaluate some $r_{n}$ for a fixed $n$, the optimal overall ratio is at most $\frac{n+1}{n} r_{n}$.

For $n=3$, maximizing the function $\hat{r}_{n}(\mathbf{s})$ can be done by hand and the maximum is $r_{3}=1.2$ for $s_{1}=s_{2}=1, s_{3}=0$. This yields an overall upper bound of $\hat{r}_{n} \leq \frac{4}{3} \cdot \frac{6}{5}=1.6$. By a computer-assisted proof we have shown that $\hat{r}_{4}=(\sqrt{7}+1) / 3 \approx 1.215$, yielding an overall upper bound of $\hat{r}_{n} \leq \frac{5}{4} \hat{r}_{4}=\frac{5}{12}(\sqrt{7}+1) \approx 1.52$.

Conclusions. Similar methods can be used to analyze other semi-online restrictions, their combinations and inexact versions, or give formulas for the approximation ratios for more machines. This becomes a somewhat mechanical exercise; we have not found any surprising phenomenon in the cases we have examined so far.

It would be interesting, and it seems hard to us but not impossible, to determine the exact overall approximation ratios for the basic restrictions.

\section{References}

[1] A. Borodin, S. Irani, P. Raghavan, and B. Schieber. Competitive paging with locality of reference. J. Comput. Systems Sci., 50:244-258, 1995.

[2] B. Chen, A. van Vliet, and G. J. Woeginger. An optimal algorithm for preemptive on-line scheduling. Oper. Res. Lett., 18:127-131, 1995.

[3] D. Du. Optimal preemptive semi-online scheduling on two uniform processors. Inform. Process. Lett., 92(5):219-223, 2004.

[4] T. Ebenlendr, W. Jawor, and J. Sgall. Preemptive online scheduling: Optimal algorithms for all speeds. In Proc. 13th European Symp. on Algorithms (ESA), volume 4168 of Lecture Notes in Comput. Sci., pages 327-339. Springer, 2006.

[5] T. Ebenlendr and J. Sgall. Optimal and online preemptive scheduling on uniformly related machines. In Proc. 21st Symp. on Theoretical Aspects of Computer Science (STACS), volume 2996 of Lecture Notes in Comput. Sci., pages 199-210. Springer, 2004.

[6] L. Epstein and L. M. Favrholdt. Optimal preemptive semi-online scheduling to minimize makespan on two related machines. Oper. Res. Lett., 30:269-275, 2002.

[7] L. Epstein and J. Sgall. A lower bound for on-line scheduling on uniformly related machines. Oper. Res. Lett., 26(1):17-22, 2000.

[8] T. F. Gonzales and S. Sahni. Preemptive scheduling of uniform processor systems. J. ACM, 25:92-101, 1978.

[9] Y. He and G. Zhang. Semi on-line scheduling on two identical machines. Computing, 62(3):179-187, 1999.

[10] E. Horwath, E. C. Lam, and R. Sethi. A level algorithm for preemptive scheduling. J. ACM, 24:32-43, 1977.

[11] Y. Jiang and Y. He. Optimal semi-online algorithms for preemptive scheduling problems with inexact partial information. Theoret. Comput. Sci., 44(7-8):571-590, 2007.

[12] H. Kellerer, V. Kotov, M. G. Speranza, and Z. Tuza. Semi on-line algorithms for the partition problem. Oper. Res. Lett., 21:235-242, 1997.

[13] S. Seiden, J. Sgall, and G. J. Woeginger. Semi-online scheduling with decreasing job sizes. Oper. Res. Lett., 27:215-221, 2000.

[14] Z. Tan and Y. He. Semi-on-line problems on two identical machines with combined partial information. Oper. Res. Lett., 30:408-414, 2002.

[15] Z. Tan and Y. He. Semi-online scheduling problems on two identical machines with inexact partial information. Theoret. Comput. Sci., 377(1-3):110-125, 2007.

This work is licensed under the Creative Commons Attribution-NoDerivs License. To view a copy of this license, visit http://creativecommons.org/licenses/by-nd/3.0/. 